\title{
Role of Arachidonic Acid-Derived Cyp450 Metabolites In Acute Kidney Injury
}

\author{
Ao Ding\#, Ye Zhu\#, You Zuo, Si-Rui Liu, Xuehong Li and Cheng Wang* \\ Department of Nephrology, $5^{\text {th }}$ Affiliated Hospital of Sun Yat-Sen University, China \\ \# These authors contributed equally \\ *Corresponding author: Cheng Wang, MD, Department of Nephrology, $5^{\text {th }}$ Affiliated Hospital of Sun Yat-Sen University, \\ China
}

\section{ARTICLE INFO}

Received: 幽 March 09, 2020

Published: 絊 March 17, 2020

Citation: Ao Ding, Ye Zhu, You Zuo, Si-Rui Liu, Cheng Wang, et al., Role of Arachidonic Acid-Derived Cyp450 Metabolites In Acute Kidney Injury. Biomed J Sci \& Tech Res 26(4)-2020. BJSTR. MS.ID.004384.

\begin{abstract}
Acute Kidney Injury (AKI) remarkblely affect public health and need higher healthcare costs, which is life-threatening and characterized of acute decline with function. Arachidonic Acid (AA), the precursor for synthesis of various biologically active molecules and be involved in regulation of homeostasis and metabolism. Recent studies have shown that the AA cytochrome P450 (CYP450) pathway contribute to the development of AKI by EETs (the product of catalyzing epoxidation reactions), 20-HETE (the product of omega-hydroxylation reactions) and maybe potent key for the prevention and treatment of AKI. This review summarizes recent advances in AA metabolites in AKI.
\end{abstract}

Keywords: Arachidonic Acid; Epoxyeicosatrienoic Acids; 20-Hydroxyeicosatetraenoic Acid; Acute Kidney Injury (AKI); Cytochrome P450 (CYP450)

\section{Introduction}

Acute kidney injury (AKI) is a common clinical syndrome that results in the accumulation of serum creatinine and urea nitrogen, mostly caused by Ischemia/Reperfusion (I/R), and its morbidity and mortality have increased within the last years [1-3]. Recent preclinical studies suggest that Arachidonic Acid (AA) metabolites generated by Cytochrome P450 (CYP) enzymes play an important role in the development of I/R-induced organ injury, expecially the heart and brain $[4,5]$. This review provide promising target for treatment with AKI by investigating the role of AA derivatives.

\section{Mechanism of AA Derivatives}

The formation of 20-Hydroxeicosatetraenoic Acid (20-HETE) is mainly generated by CYP4A and CYP4F families, while a set of regio- and stereoisomeric Epoxyeicosatrienoic Acids (EETs) is produced through the action of CYP 2J and 2C enzymes [6]. EETs are produced in vascular endothelial cells and activate calcium-activated potassium (BK) channels in the underlying vascular smooth muscle cells, eventually leading to vasorelaxation [7-9]. While 20-
HETE, which is produced by renal vascular smooth muscle cells, acts as a potent vasoconstrictor of small arteries and arterioles $(<100 \mu \mathrm{m})$ such as renal interlobular and afferent arterioles [6]. The proportional distribution of 20-HETE and EETs could potentially influence fluid homeostasis and vascular tone and become a key determinant for controlling blood pressure [10]. Apart from the regulation of renal vascular response, the role of CYP-dependent AA metabolites in the control of sodium excretion has emerged as a dynamic new field [11]. 20-HETE and EETs are formed in different segments of the nephron and mediate tubular function with the net effect of inhibiting sodium reabsorption [12,13]. Overall, in the kidney, the eicosanoids (20-HETE and EETs) have concordant effects on sodium reabsorption but contradictive effects on vascular reactivity [14].

\section{Impact of AA Derivatives in Aki}

There are few reports that have addressed the role of 20-HETE on renal I/R-injury. In vitro experiments showed that 20-HETE overproduction can significantly exacerbate the cytotoxic and 
pro-apoptotic effects of chemical hypoxia on cultured renal tubular epithelial cells [15]. Moreover, Hoff et al. concluded that ischemia-induced 20-HETE generation and action are primarily responsible for initiating the pathophysiological cascade leading to I/R-induced kidney injury [16]. This conclusion is also in line with a recent clinical study on renal transplantation showing that the extent of 20-HETE released within the first $5 \mathrm{~min}$ of allograft reperfusion is a negative predictor of post-transplant allograft function [17]. In contrast, there is definite evidence to indicate that EETs have significant effect of decreasing inflammatory cell infiltration, limiting of leukocyte adhesion, reducing apoptosis and vasodilatation $[18,19]$. Accordingly, Hoff et al, also suggested that ischemia could cause the imbalance between 20-HETE and EETs, while they play opposite role in kidney. Administration of exogenous EET analogue exert better protective impact and compensate the lack of endogenous EET in ischemia-induced AKI [20].

\section{Conclusion}

This review focus on the current evidence about the critical effect of AA metabolites in AKI and provide efficacious therapeutic targets in AKI. Considering all the evidence, ischemia-induced AKI leads to the imbalance between 20-HETE and EETs, overproduced 20-HETE and the lack of endogenous EET, which support that AA metabolites play a critical role in regulating the pathophysiology processes of AKI. Thus, targeting the formation and action of CYP-dependent eicosanoids will probably have beneficial effects on the prevention of ischemic AKI in clinical setting.

\section{References}

1. Chen J, Zeng H, Ouyang X, Zhu M, Huang Q, et al. (2020) The incidence, risk factors, and long-term outcomes of acute kidney injury in hospitalized diabetic ketoacidosis patients. BMC Nephrol 21(1): 48.

2. James MT, Bhatt M, Pannu N, Tonelli M (2020) Long-term outcomes of acute kidney injury and strategies for improved care. Nat Rev Nephrol.

3. Oh DJ (2020) A long journey for acute kidney injury biomarkers. Ren Fail 42(1): 154-165.

4. Williams JM, Murphy S, Burke M, Roman RJ (2010) 20-hydroxyeicosatetraeonic acid: A new target for the treatment of hypertension[J]. J Cardiovasc Pharmacol 56(4): 336-344.

5. Theken KN, Schuck RN, Edin ML, Tran B, Ellis K, et al. (2012) Evaluation of cytochrome P450-derived eicosanoids in humans with stable

ISSN: 2574-1241

DOI: 10.26717/BJSTR.2020.26.004384

Cheng Wang. Biomed J Sci \& Tech Res

Submission Link: https://biomedres.us/submit-manuscript.php
This work is licensed under Creative

Commons Attribution 4.0 License

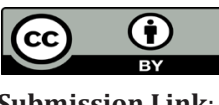

atherosclerotic cardiovascular disease. Atherosclerosis 222(2): 530536.

6. Roman RJ (2002) P-450 metabolites of arachidonic acid in the control of cardiovascular function. Physiol Rev 82(1): 131-185

7. Fan F, Ge Y, Lv W, Elliott MR, Muroya Y, et al. (2016) Molecular mechanisms and cell signaling of 20-hydroxyeicosatetraenoic acid in vascular pathophysiology. Front Biosci (Landmark Ed) 21: 1427-1463.

8. Garcia V, Schwartzman ML (2017) Recent developments on the vascular effects of 20-hydroxyeicosatetraenoic acid[J]. Curr Opin Nephrol Hypertens 26(2): 74-82.

9. Roman RJ, Akbulut T, Park F, Regner KR (2011) 20-HETE in acute kidney injury. Kidney Int 79(1): 10-13.

10. Fan F, Roman RJ (2017) Effect of Cytochrome P450 Metabolites of Arachidonic Acid in Nephrology[J]. J Am Soc Nephrol 28(10): 28452855.

11. Maier KG, Roman RJ (2001) Cytochrome P450 metabolites of arachidonic acid in the control of renal function[J]. Curr Opin Nephrol Hypertens 10(1): 81-87.

12. McGiff JC and Quilley J (1999) 20-HETE and the kidney: Resolution of old problems and new beginnings[J]. Am J Physiol 277(3): R607-R623.

13. Schwartzman ML, da Silva JL, Lin F (1996) Cytochrome P450 4A expression and arachidonic acid omega-hydroxylation in the kidney of the spontaneously hypertensive rat. Nephron 73(4): 652-663.

14. Liu X, Wu J, Liu H, Lai G, Zhao Y (2012) Disturbed ratio of renal 20-HETE/ EETs is involved in androgen-induced hypertension in cytochrome P450 4F2 transgenic mice. Gene 505(2): 352-359.

15. Nilakantan V, Maenpaa C, Jia G, Roman RJ, Park F (2008) 20-HETEmediated cytotoxicity and apoptosis in ischemic kidney epithelial cells[J]. Am J Physiol Renal Physiol 294(3): F562-F570.

16. Hoff U, Lukitsch I, Chaykovska L, Ladwig M, Arnold C, et al. (2011) Inhibition of 20-HETE synthesis and action protects the kidney from ischemia/reperfusion injury. Kidney Int 79(1): 57-65.

17. Dolegowska B, Blogowski W, Domanski L (2009) Is it possible to predict the early post-transplant allograft function using 20-HETE measurements? A preliminary report. Transpl Int 22(5): 546-553.

18. Deng BQ, Luo Y, Kang X, Li CB, Morisseau C, et al. (2017) Epoxide metabolites of arachidonate and docosahexaenoate function conversely in acute kidney injury involved in GSK3beta signaling[J]. Proc Natl Acad Sci U S A 114(47): 12608-12613.

19. Zhu Y, Blum M, Hoff U, Wesser T, Fechner M, et al. (2016) Renal Ischemia/ Reperfusion Injury in Soluble Epoxide Hydrolase-Deficient Mice[J]. PLoS One 11(1): e0145645.

20. Hoff U, Bubalo G, Fechner M, Blum M, Zhu Y, et al. (2019) A synthetic epoxyeicosatrienoic acid analogue prevents the initiation of ischemic acute kidney injury[J]. Acta Physiol (Oxf) 227(2): e13297.

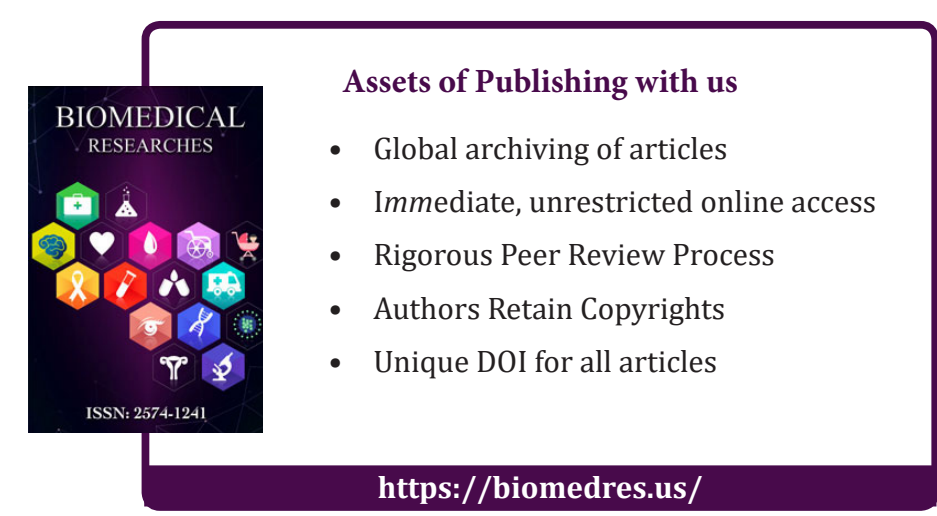

